\section{Saussurea species in Indian Himalayan Region: diversity, distribution and indigenous uses}

\author{
Jitendra S. Butola' and Sher S. Samant ${ }^{2}$ \\ ${ }^{1}$ G.B. Pant Institute of Himalayan \\ Environment and Development, Kosi- \\ Katarmal, Almora, Uttarakhand, India; \\ ${ }^{2}$ G.B. Pant Institute of Himalayan \\ Environment and Development, Mohal- \\ Kullu, Himachal Pradesh, India
}

\section{Abstract}

In spite of the high economic value of the Saussurea species in the Indian Himalayan Region (IHR), the potential of most of the species is yet to be investigated. Therefore, an attempt has been made to study the diversity, distribution, habitat preference, nativity, endemism, status and indigenous uses of Saussurea species in the IHR. A total of 62 species were recorded from the IHR; of these, 37 species were native to the Himalayan region, 8 were endemic and 21 were near endemic to the IHR. Twenty-seven of the 28 species that were known to have indigenous uses also had medicinal value and are used for the treatment of various diseases/ailments. Many species, e.g., $S$. affinis (Ganga Mula*), S. auriculata (Pachak Kut), S. bracteata (Prerak Mul), S. costus (Kuth), S. gossypiphora (Kasturi Kamal) and $S$. obvallata (Brahm Kamal), have multiple uses. The genus showed high habitat specificity in that 16 species were recorded to be restricted to one or two habitats only. As many as 44 species were identified as rare in the study region. Considering the high industrial demand for raw materials and the endangered status of $S$. costus, S. gossypiphora, S. obvallata and $S$. simpsoniana (Fen Kamal), these species should be prioritized for conservation (in situ and ex situ) throughout the IHR. Population assessment of the rare-endangered, native, endemic and economically important species using standard ecological methods has been suggested for the quantification of the existing stock of these species in their natural habitats. Further, phytochemical investigations for the identification of active ingredients are suggested. Propagation and cultivation techniques are lacking for most of the species of Saussurea except for $S$. costus, S. obvallata and S. medusa (Snow Lotus). Furthermore, the native communities need to be sensitized to the sustainable use and conservation value of the species in this genus.

*Local names given in parentheses throughout are in the Pahari language, spoken by native communities in the Himalayan region.

\section{Introduction}

It is well known that the Indian Himalayan Region (IHR) has a great range of plant diversity. The region alone supports about 18,440 species of plants (Angiosperms: 8,000 spp., Gymnosperm: 44 spp., Pteridophytes: 600 spp., Bryophytes: 1,736 spp., Lichens: 1,159 spp. and Fungi: 6,900 spp.). ${ }^{1}$ According to Samant et al., ${ }^{2}$ out of the total species of vascular plants, 1,748 species are medicinal. Asteraceae is the fourth largest family of vascular plants, comprising over 30,000 species (1,100 genera), and is distributed almost worldwide. In India, the family is estimated to have about 900 species under 167 genera. ${ }^{3,4}$ Plants of the family are perennial and most species are herbaceous in nature, with trees and shrubs representing only about $2 \%$ of the total.

Saussurea is named after Horace Benedict de Saussure (1740-1799), a Swiss philosopher. It is an important genus of the family comprising an estimated 410 species, native to cool temperate and arctic regions of Asia, Europe, and North America, with the highest diversity in alpine habitats in the Himalayas and Central Asia. Of the estimated species, 61 are found in India. ${ }^{3,5,6}$ The genus is quite varied and is well known to have medicinal, religious and other economic values, i.e. food, flavoring material, rubber, oil, insecticides, dye, ornamental value, etc.

Plants of the Saussurea genus range in height from the $5-10 \mathrm{~cm}$ tall dwarf alpine species to tall, thistle-like plants up to $3 \mathrm{~m}$ tall. The leaves are produced in a dense basal rosette, and spiral up the flowering stem. The flowers form in a dense head of small capitula, often completely surrounded in dense white to purple woolly hairs; the individual florets are also white to purple. The woolly hairs in high altitude species of the family are the densest, and aid in thermoregulation of the flowers, minimizing frost damage at night, and also preventing ultraviolet light damage from the intense high altitude sunlight. ${ }^{?}$

$S$. costus (Kuth, Figure 1A), native to the Himalayan Region, ${ }^{2}$ is one of the most commercially viable species of this genus and is in high demand for treating many diseases like bronchial asthma, rheumatism, cholera, jaundice, leprosy, etc. ${ }^{8}$ Besides this species, $S$. gossypiphora (Kasturi Kamal, Figure 1B) and S. obvallata (Brahm Kamal, Figure 1C) are very popular for medicinal and religious purposes in the IHR. A review of literature reveals that the information available for a few species of Saussurea in the region is fragmentary.

To our knowledge, there is hardly any documentation available which contains comprehensive information on the diversity, distribution, habitat preference, nativity, endemism, status and indigenous uses of the species of
Correspondence: Jitendra S. Butola, G.B. Pant Institute of Himalayan Environment and Development, Kosi-Katarmal, Almora-263 643, Uttarakhand, India. E-mail or butolajs@rediffmail.com or drbutolajs@yahoo.in

Key words: Saussurea, diversity, distribution, indigenous uses, habitat preference, nativity, endemism, status, conservation.

Acknowledgments: the authors are thankful to Dr. U. Dhar, Director, G.B. Pant Institute of Himalayan Environment and Development, KosiKatarmal, Almora, Uttarakhand, India for providing necessary facilities and consistent support. JS Butola wishes to thank Dr. Ashok Singh for helping in the literature survey.

Received for publication: 27 January 2010

Revision received: 17 February 2010.

Accepted for publication: 3 March 2010.

This work is licensed under a Creative Commons Attribution 3.0 License (by-nc 3.0).

(C) Copyright J.S. Butola and S.S. Samant, 2010 Licensee PAGEPress, Italy

International Journal of Plant Biology 2010; 1:e9 doi:10.4081/pb.2010.e9

Saussurea in the IHR. In view of the economic importance of the genus for the inhabitants of the region, this study was undertaken to: i) examine the diversity, distribution pattern and indigenous uses of the species of Saussurea in the IHR; ii) assess and analyze species for their habitat preference, nativity, endemism and status; and iii) suggest appropriate strategies for the conservation management of the species.

\section{Materials and Methods}

\section{Survey, sampling and identification of the species}

While exploring the floristic diversity of the West Himalayas between 1993 and 2003 and the North-West Himalayas between 2004 and 2006, we recorded observations on distribution, altitudinal range, habit and habitat of the Saussurea species. Knowledge of indigenous uses was gathered through interviews with local inhabitants. This was augmented by an extensive survey of literature on the species of Saussurea occurring in the IHR., ${ }^{2,625}$

\section{Determination of nativity,} endemism and IUCN status of the species

Nativity of the species has been identified $d^{2,26}$ and determination of endemism was based on 
the distribution range of the species. ${ }^{2,27}$ The species restricted to the IHR have been considered as endemic whereas those with extended distribution to neighboring countries/states have been considered as near endemic. IUCN status of the species was determined based on occurrence of the species in natural habitats.

\section{Review of propagation and cultiva- tion}

An extensive survey of literature was carried out to determine the current status of propagation and cultivation of Saussurea species in the IHR. For the review of literature, traditional libraries at a variety of different institutions, as well as various websites, were utilized. ${ }^{828-42}$

\section{Results and Discussion}

\section{Diversity, distribution and habitat preference}

In the present study, 62 species of Saussurea were recorded from the IHR. All the species were herbaceous in nature and distributed between $550 \mathrm{~m}$ and $5,700 \mathrm{~m}$. The greatest species diversity (33 spp.) was recorded in the zone between 3,500 $\mathrm{m}$ and 5,000 $\mathrm{m}$ (Figure 2). This high species diversity may be due to varied soil, climate and geography of the zone, which gives rise to many micro and macro habitats. ${ }^{22}$

The Northeast region of the Himalayas, particularly Sikkim, contains the greatest number of species of Saussurea (32 spp.), which is among the dominant plant genera in the state. ${ }^{43}$ In the present study, Saussurea was found within 15 habitats: shady/moist, alpine meadows/slopes, shrubberies, open grassy slope, shady rock/boulders, roadside/waste places, cultivated area/agricultural lands, dry places, rocky slopes, snowline/glacier slopes, forest, riverine, alpine screes, flooded areas and saline alkali lands (Table 1). Maximum species diversity was in alpine meadows/slopes (55 spp.), followed by shady/moist places (32 spp.), alpine screes (24 spp.) and forest (23 spp.) habitats. Sixteen species were restricted to one or two habitats only.

\section{Native and endemic species}

Of the total Saussurea species identified, 37 species were determined to be native to the Himalayan region and the remaining 25 species were non-native (Table 1). Among the native species, 8 species (S. atkinsoni, S. ceratocarpa, S. clarkei, S. costus, S. laneana, S. obscura, S. pantlingiana and S. sudhanshui) were endemic to the IHR, while 21 other species ( $S$. abnormis, $S$. albescens, $S$. andersonii, $S$. andryaloides, $S$. auriculata, $S$. candol-

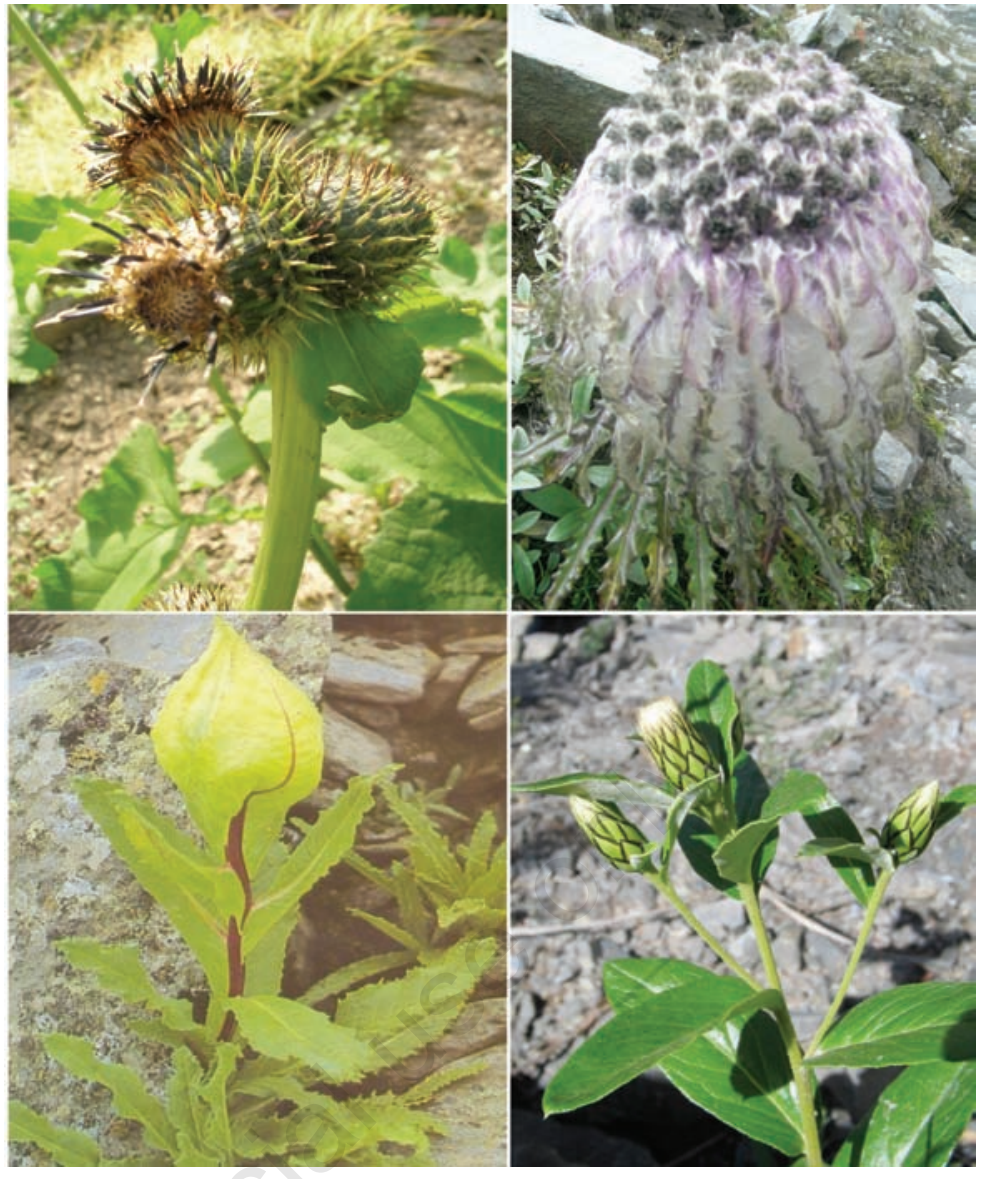

Figure 1. (A) Saussurea costus (photo: Dr. J.S. Butola); (B) Saussurea gossypiphora (photo: Dr. R.S. Chauhan); (C) Saussurea obvallata (photo: Dr. S.S. Samant) and (D) Saussurea heteromalla (photo: Dr. Manohar Lal).

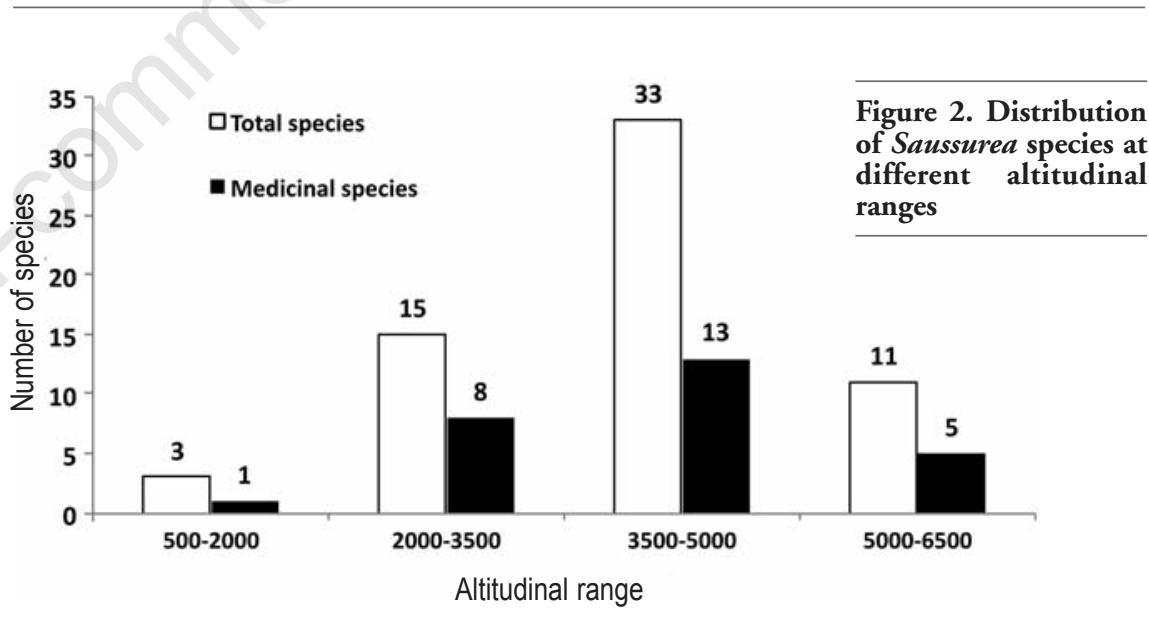

leana, S. conica, S. gilesii, S. graminifolia, $S$. heteromalla (Kaliziri, Figure 1D), S. jacea, $S$. nimborum, S. nishiokae, S. piptathera, S. polystichoides, $S$. roylei, S. sughoo, $S$. taraxacifolia, S. thomsonii, S. tridactyla and S. yakla) had extended distribution to neighboring countries like Pakistan, Afghanistan, Bhutan, Tibet and Nepal, and were thus identified as near endemic. Endemic and habitat-specific species are generally considered more prone to extinction than widespread habitat generalists. Among the dominant high altitude genera in the Himalaya, Saussurea is the second largest genus with a preponderance of endemic species (35 spp.), ${ }^{44}$ suggesting that there is great value in conservation management of the species in this genus.

\section{Indigenous uses}

Proper documentation and compilation of indigenous knowledge of a species helps to identify the potential and conservation value of the species and the document can be used as a tool to transfer this knowledge from genera- 
Table 1. Diversity, distribution, habitat preference, status and indigenous uses of the species of Saussurea in the Indian Himalayan Region.

\begin{tabular}{|c|c|c|c|c|c|c|c|}
\hline Taxa & LN/VN & $\begin{array}{l}\text { Altitudinal } \\
\text { range (m) }\end{array}$ & Habitat(s) & Nativity & Distribution & Status & $\begin{array}{l}\text { Indigenous } \\
\text { uses }\end{array}$ \\
\hline **Saussurea abnormis Lipsch. & - & $3500-4600$ & 2,13 & Ind & $\begin{array}{l}\text { Tibet, India } \\
\text { (Chamoli Garhwal, } \\
\text { Uttar Pradesh), Nepal }\end{array}$ & Rare & Medicinal \\
\hline S. affinis Spreng. & Ganga Mula & 3300 & $2,3,6,7,11,12$ & As Trop & $\begin{array}{l}\text { E Himalaya (Assam), } \\
\text { E Asia, China, Japan }\end{array}$ & Occ & $\begin{array}{l}\text { Leaves and young shoots } \\
\text { are edible. The juice of root } \\
\text { is one of the ingredients used } \\
\text { in medicines for women's diseases. }\end{array}$ \\
\hline **S. albescens (DC.) Sch.-Bip. & $\begin{array}{l}\text { Pirya, } \\
\text { Bacha-Shang, } \\
\text { Drapada }\end{array}$ & $1500-3000$ & 2,4,6,8,11, & Reg Himal & $\begin{array}{l}\text { NE Afghanistan, NW India, } \\
\text { (Kashmir, Himachal } \\
\text { Pradesh), CW Nepal, } \\
\text { N Pakistan }\end{array}$ & Unco & $\begin{array}{l}\text { Heads soaked in water and decant } \\
\text { is taken to relieve bronchitis. } \\
\text { Leaves for diuretic activities. }\end{array}$ \\
\hline${ }^{* * S}$. andersonii $\mathrm{Cl}$. & - & $3500-4200$ & 1,2 & Reg Himal & $\begin{array}{l}\text { E Himalaya (Sikkim), } \\
\text { S Tibet }\end{array}$ & Rare & Medicinal \\
\hline${ }^{* *}$ S. andryaloides (DC.) Sch.-Bip. & & $4400-4700$ & 9 & Reg Himal & $\begin{array}{l}\text { W Himalaya (Kashmir), } \\
\text { Pakistan }\end{array}$ & Rare & - \\
\hline S. aster Hemsl. & - & $3900-5400$ & $1,2,13$ & Tibet & $\begin{array}{l}\text { W Himalaya } \\
\text { (Ladakh, Himachal } \\
\text { Pradesh), China }\end{array}$ & Rare & - \\
\hline *S. atkinsoni $\mathrm{Cl}$. & - & $3300-3900$ & 2,10 & Reg Himal & $\begin{array}{l}\text { W Himalaya (Himachal } \\
\text { Pradesh, } \\
\text { Ladakh, Uttarakhand) }\end{array}$ & Rare & Medicinal \\
\hline $\begin{array}{l}{ }^{* *} \text { S. auriculata } \\
\text { (Spreng. ex DC.) Schi.-Bip. }\end{array}$ & $\begin{array}{l}\text { Thimra, } \\
\text { Nurya, } \\
\text { Pachak Kut }\end{array}$ & $3000-3800$ & $1,2,3,5,11$ & Tibet & $\begin{array}{l}\text { Bhutan, India (Uttar } \\
\text { Pradesh, Uttar } \\
\text { Kashi, Harki Dun, Kashmir, } \\
\text { Sikkim, Himachal Pradesh), } \\
\text { Nepal, N Pakistan, S Tibet }\end{array}$ & Rare & $\begin{array}{l}\text { Leaves are edible and are considered } \\
\text { purgative and antisyphilitic. } \\
\text { Leaf paste of the plant and Betula } \\
\text { utilis used externally in venereal } \\
\text { diseases. Roots are a } \\
\text { substitute forKuth. }\end{array}$ \\
\hline S. bodinieri Lev. & - & $3200-4700$ & $2,4,13$ & $\begin{array}{l}\text { Ind Or } \\
\text { China }\end{array}$ & $\begin{array}{l}\text { China, Bhutan, N Myanmar, } \\
\text { E Nepal, } \\
\text { SE Tibet, India (Sikkim) }\end{array}$ & Occ & - \\
\hline S. bracteata Decne. & $\begin{array}{l}\text { Chhota Doda, } \\
\text { Prerak Mul }\end{array}$ & $3500-4800$ & $1,2,5,10,13$ & Reg Himal & $\begin{array}{l}\text { China, India } \\
\text { (Uttar Pradesh, Kumaon, } \\
\text { Dhauli valley, Kashmir), } \\
\text { N Pakistan, W Tibet }\end{array}$ & Rare & $\begin{array}{l}\text { Roots are stimulant and used in boils, } \\
\text { headache, cough, cold, fever and lung } \\
\text { infection. Also a good soil binder. }\end{array}$ \\
\hline **S. candolleana Wall. ex. DC. & - & $2400-4400$ & $1,2,9,11$ & Reg Himal & $\begin{array}{l}\text { Bhutan, India } \\
\text { (Uttar Pradesh, Kumaon, } \\
\text { Pindari, Himachal Pradesh, } \\
\text { Kashmir), Nepal }\end{array}$ & Rare & - \\
\hline *S. ceratocarpa Decne. & Pashka & $3500-5000$ & $1,2,11$ & Reg Himal & $\begin{array}{l}\text { Indian Himalaya } \\
\text { (Kashmir) }\end{array}$ & Occ & $\begin{array}{l}\text { Whole plant is used in colic, } \\
\text { headache, lumbar pain, menorrhea, } \\
\text { renel pain. }\end{array}$ \\
\hline *S. clarkei H. f. & - & $>2500$ & $1,2,13$ & Reg Himal & Indian Himalaya (Kashmir) & Rare & Medicinal \\
\hline **S. conica $\mathrm{Cl}$. & & $4600-5300$ & 2,4 & Reg Himal & Bhutan, India (Sikkim) & Rare & Medicinal \\
\hline *S. costus (Falc.) Lipsch. & $\begin{array}{l}\text { Kuth, Kut, } \\
\text { Kostus, Kstha }\end{array}$ & $3300-4000$ & $1,2,7$ & Reg Himal & $\begin{array}{l}\text { India (Kashmir, Lahaul } \\
\text { \& Spiti, Uttar Kashi, Barsu) }\end{array}$ & CR & $\begin{array}{l}\text { The root is used in dysentery, } \\
\text { rheumatism, skin disorder, } \\
\text { stomachache, cough, cold, cholera, } \\
\text { bronchitis, fever, edema, gas, } \\
\text { jaundice, leprosy, phlegm and skin } \\
\text { diseases, toothache, earache, ulcer, } \\
\text { bruises and cuts. Also used as a tonic, } \\
\text { carminative, stimulant, spasmodic, for } \\
\text { dyspepsia and in controlling bronchial } \\
\text { asthma. Half teaspoonful powder of } \\
\text { the root is given for arthritis for 7-15 } \\
\text { days. The aromatic root is sometimes } \\
\text { used as a spice. Also used as } \\
\text { insecticide to protect shawls and } \\
\text { woollen fabrics and an incense. The } \\
\text { root oil has a very strong odor and } \\
\text { used in high-grade perfume. The oil is } \\
\text { also employed in the preparation of } \\
\text { hair oil. Apart from medicinal } \\
\text { purposes, the upper parts of the } \\
\text { plant are used as fodder and fuel. }\end{array}$ \\
\hline
\end{tabular}


Table 1. Continued from previous page.

\begin{tabular}{|c|c|c|c|c|c|c|c|}
\hline Taxa & LN/VN & $\begin{array}{l}\text { Altitudinal } \\
\text { range (m) }\end{array}$ & Habitat(s) & Nativity & Distribution & Status & Indigenous uses \\
\hline S. crispa Vaniot. & - & $1200-1800$ & $1,2,11$ & China & $\begin{array}{l}\text { India (Himachal } \\
\text { Pradesh, Assam), Myanmar, } \\
\text { Bhutan, Nepal, Tibet, Burma, } \\
\text { SW China, Taiwan Laos, } \\
\text { Thailand, Vietnam }\end{array}$ & $\begin{array}{l}\text { Rare } \\
\text { a, }\end{array}$ & - \\
\hline S. deltoidea (DC.) Sch. Bip. & - & $800-3400$ & $2,3,4,6,11$ & Reg Himal & $\begin{array}{l}\text { Bhutan, India (Sikkim), } \\
\text { Laos, Myanmar, Nepal, } \\
\text { Pakistan, Thailand, Vietnam, } \\
\text { China, Tibet }\end{array}$ & Co & - \\
\hline S. depsangensis Pampanini & - & $4800-5400$ & 2,13 & Mont Kara & $\begin{array}{l}\text { Tibet, China, India } \\
\text { (Kashmir) }\end{array}$ & Rare & - \\
\hline S. donkiah Cl. ex Spring. & - & $4000-5000$ & 1,2 & Ind Nepal & $\begin{array}{l}\text { E Nepal, Bhutan, China, } \\
\text { India (Sikkim) }\end{array}$ & Rare & - \\
\hline S. fastuosa (Decne.) Sch.-Bip. & $\begin{array}{l}\text { Proud } \\
\text { Saw-wart }\end{array}$ & $2200-3600$ & $2,3,4,11$ & Reg Himal & $\begin{array}{l}\text { Myanmar, India (Kumaon, } \\
\text { Uttar Pradesh, Chamoli } \\
\text { Garhwal, Valley of Flower, } \\
\text { Himachal Pradesh, Sikkim), } \\
\text { Nepal, Tibet, N Burma, } \\
\text { SW China }\end{array}$ & Rare & - \\
\hline S. forrestii Diels & - & $2000-3600$ & 1,2 & China & $\begin{array}{l}\text { China, India (Sikkim), } \\
\text { C Nepal }\end{array}$ & Rare & Medicinal \\
\hline **S. gilesii Hemsley & - & $3000-4200$ & 2,4 & $\begin{array}{l}\text { Reg } \\
\text { Afghan }\end{array}$ & $\begin{array}{l}\text { NE Afghanistan, India } \\
\text { (Kashmir), N Pakistan }\end{array}$ & Rare & - \\
\hline S. glabrata (DC.) C. Shih & - & $3500-4000$ & 1 & Sibir & NW India, Siberia & Rare & - \\
\hline S. glacialis Herder. & - & $>2500$ & $1,4,13$ & Turk & $\begin{array}{l}\text { Kazakstan, Kyrgyzstan, } \\
\text { Mongolia, Russia (Siberia), } \\
\text { Tajikistan NE Afghanistan, } \\
\text { N Pakistan, India (Jammu } \\
\text { \& Kashmir, Ladakh, } \\
\text { Thang Lang La) }\end{array}$ & Occ & $\begin{array}{l}\text { Amchi use its leaves and flowers in } \\
\text { liver, throat and heart troubles. } \\
\text { It is also used in mental disorder. }\end{array}$ \\
\hline S. glanduligera Schi.-Bip. & - & $3000-4500$ & 1,2 & Reg Himal & $\begin{array}{l}\text { India (Kashmir, Himachal } \\
\text { Pradesh, Spiti), } \\
\text { N Pakistan, China }\end{array}$ & Rare & - \\
\hline $\begin{array}{l}\text { S. gnaphalodes (Royle) } \\
\text { Sch.-Bip. }\end{array}$ & $\begin{array}{l}\text { Yuliang, } \\
\text { Ravi Basin } \\
\text { Gugi }\end{array}$ & $4000-4500$ & $1,2,3$ & Reg Himal & $\begin{array}{l}\text { NE Afghanistan, India } \\
\text { (Jammu \& Kashmir, Ladakh, } \\
\text { Thang Lang La, Himachal } \\
\text { Pradesh), Tibet, Nepal, } \\
\text { W Pakistan, SW China }\end{array}$ & Occ & $\begin{array}{l}\text { Used for kidney problems, } \\
\text { cough and cold. Aerial parts } \\
\text { dried and pulverized and } 1 / 2 \\
\text { teaspoonful of powder given } 3 \\
\text { times a day for } 5 \text { - } 15 \text { days to } \\
\text { cure backache, pulmonary affections } \\
\text { and also for purification of blood. }\end{array}$ \\
\hline S. gossypiphora D. Don & $\begin{array}{l}\text { Ghuggi Badshah } \\
\text { Bhutkesh, } \\
\text { Kasturi Kamal, } \\
\text { Fini Kawal }\end{array}$ & 1, 3500-5700 & $1,2,9,13$ & Reg Himal & $\begin{array}{l}\text { Bhutan, Nepal, India } \\
\text { (Himachal Pradesh, } \\
\text { Lahaul \& Spiti, Ladakh, } \\
\text { Sikkim, Garhwal), S Tibet, } \\
\text { SW China }\end{array}$ & CR & $\begin{array}{l}\text { The plant is } \\
\text { offered for worship at hill shrines } \\
\text { and to guard against evil spirits. } \\
\text { It is reputed to possess som } \\
\text { medicinal properties, e.g. } \\
\text { gynecological disorders, menstrual } \\
\text { disorders and hysteria. Roots yield } \\
\text { essential oil used in perfumery. }\end{array}$ \\
\hline **S. graminifolia Wall. & Ghoogee & $3500-5600$ & $2,7,9,13$ & Reg Himal & $\begin{array}{l}\text { Bhutan, India } \\
\text { (Uttar Pradesh, Pindari, } \\
\text { Furkia, Kumaon, Kashmir, } \\
\text { Sikkim), Nepal, S Tibet }\end{array}$ & Rare & $\begin{array}{l}\text { The entire plant is used in Tibetan } \\
\text { medicine. It has a sour and sweet } \\
\text { taste with heating potency. } \\
\text { Antitussive, aphrodisiac, blood } \\
\text { purifier and emmenagogue, it is used } \\
\text { in the treatment of coughing due to } \\
\text { loss of potency in the spleen, } \\
\text { irregular menses, semina/vaginal } \\
\text { discharge, excessive bleeding from } \\
\text { the womb and pain at the waist due to } \\
\text { a loss of renal potency. }\end{array}$ \\
\hline
\end{tabular}


Table 1. Continued from previous page.

\begin{tabular}{|c|c|c|c|c|c|c|c|}
\hline Taxa & LN/VN & $\begin{array}{l}\text { Altitudinal } \\
\text { range (m) }\end{array}$ & Habitat(s) & Nativity & Distribution & Status & Indigenous uses \\
\hline $\begin{array}{l}\text { **S. heteromalla } \\
\text { (D. Don) Hand.-Mazz. }\end{array}$ & $\begin{array}{l}\text { Batula, } \\
\text { Murang, } \\
\text { Kaliziri }\end{array}$ & $550-4000$ & $2,6,7,9,11$ & Reg Himal & $\begin{array}{l}\text { NE Afghanistan, Bhutan, } \\
\text { India (Uttar Pradesh, } \\
\text { Uttar Kashi, Sankari, } \\
\text { Kashmir, Himachal } \\
\text { Pradesh), Nepal, N Pakistan }\end{array}$ & Co & $\begin{array}{l}\text { Leaf paste with mustard oil massaged } \\
\text { on leucoderma and wounds. Root } \\
\text { extract taken for fever and colic. } \\
\text { The seeds are carminative and } \\
\text { used for horse-bites. }\end{array}$ \\
\hline S. hieracioides Hk. & - & $3700-4950$ & $1,2,13$ & Reg Himal & $\begin{array}{l}\text { Bhutan, Nepal, India } \\
\text { (Sikkim), E Tibet, S China }\end{array}$ & Occ & - \\
\hline S. hookeri $\mathrm{Cl}$. & - & $4350-5100$ & $1,2,11$ & Reg Himal & $\begin{array}{l}\text { Bhutan, India (Kumaon, } \\
\text { Kashmir, Sikkim, Lachung), } \\
\text { E \& S Tibet, SW China }\end{array}$ & Rare & - \\
\hline **S. jacea (Klotz.) Cl. & - & $3000-4200$ & $1,2,5$ & Reg Himal & $\begin{array}{l}\text { NE Afghanistan, W Tibet, } \\
\text { India (Jammu \& Kashmir, } \\
\text { Ladakh, Dras, Himachal } \\
\text { Pradesh), N Pakistan }\end{array}$ & Rare & - \\
\hline S. katochaete Maxim. & - & $2200-4700$ & $4,11,14$ & China & $\begin{array}{l}\text { China, Bhutan, Tibet, } \\
\text { India (Sikkim) }\end{array}$ & Rare & - \\
\hline *S. laneana W.W. Sm. & - & $3300-4200$ & $1,2,11$ & Ind Or & Indian Himalaya (Sikkim) & Rare & Medicinal \\
\hline $\begin{array}{l}\text { S. leontodontoides (DC.) } \\
\text { Sch.-Bip. }\end{array}$ & - & $3500-4500$ & $1,2,13$ & Reg Himal & $\begin{array}{l}\text { India (Uttar Pradesh, } \\
\text { Chamoli Garhwal, } \\
\text { Amritganga Valley, } \\
\text { Kashmir, Himachal } \\
\text { Pradesh, Sikkim), } \\
\text { Nepal, Tibet, C China }\end{array}$ & & - \\
\hline S. medusa Maxim. & Snow Lotus & $3000-5600$ & $2,9,13$ & $\begin{array}{l}\text { China } \\
\text { Mongolia }\end{array}$ & $\begin{array}{l}\text { China, India (Kashmir), } \\
\text { Nepal, E Tibet, N Pakistan, }\end{array}$ & Rare & $\begin{array}{l}\text { The whole herb is used in traditional } \\
\text { Chinese and Tibetan medicine for the } \\
\text { treatment of headache, high blood } \\
\text { pressure and to regulate menstrual } \\
\text { cycles and treat menstrual problems. } \\
\text { Also used as tonic for weakness and } \\
\text { remedy for arthritis. }\end{array}$ \\
\hline **S. nimborum W.W. Sm. & - & $4500-5000$ & $2,4,12$ & Ind Or & $\begin{array}{l}\text { Bhutan, India } \\
\text { (Sikkim, Kashmir), E Tibet }\end{array}$ & Occ & - \\
\hline S. nepalensis Spreng. & $\begin{array}{l}\text { Nepal } \\
\text { Saw-wort }\end{array}$ & $3200-4900$ & $1,2,3,9,11,13$ & Nepal & $\begin{array}{l}\text { Bhutan, Nepal, India } \\
\text { (Sikkim), S Tibet, China }\end{array}$ & Rare & - \\
\hline **S. nishiokae Kitam. & - & $4500-4900$ & $1,2,11$ & $\begin{array}{l}\text { Bhutan } \\
\text { Nepal }\end{array}$ & $\begin{array}{l}\text { Bhutan, India (Sikkim), } \\
\text { Nepal }\end{array}$ & Rare & - \\
\hline *S. obscura Lipsch. & - & $3600-4800$ & $1,2,4$ & Reg Himal & Indian Himalaya (Sikkim) & Rare & - \\
\hline S. obvallata (DC.) Edgew. & $\begin{array}{l}\text { Brahm Kamal, } \\
\text { Kanwal, Birm } \\
\text { Kanwal }\end{array}$ & $3000-4800$ & $2,3,5,9,10,13$ & Reg Himal & $\begin{array}{l}\text { Myanmar, Bhutan, India } \\
\text { (Uttar Pradesh, Chamoli } \\
\text { Garhwal, Hemkund, } \\
\text { Kashmir, Sikkim), E Tibet, } \\
\text { Nepal, Pakistan }\end{array}$ & EN & $\begin{array}{l}\text { The flowers, rhizome and leaves are } \\
\text { used for various traditional, } \\
\text { religious, medicinal and ornamental } \\
\text { purposes. The roots are applied to } \\
\text { bruises, boils, wounds and cuts and } \\
\text { used as a nerve tonic and powerful } \\
\text { antiseptic. It is used in several } \\
\text { Tibetan medicines and in the } \\
\text { treatment of paralysis of the limbs } \\
\text { and cerebral ischemia. }\end{array}$ \\
\hline S. pachyneura Franch. & - & $3900-5300$ & $1,2,13$ & $\begin{array}{l}\text { China } \\
\text { Occi }\end{array}$ & $\begin{array}{l}\text { India (Sikkim), } \\
\text { Myanmar, Bhutan, E Nepal, } \\
\text { Burma, SW China }\end{array}$ & Rare & - \\
\hline${ }^{*}$ S. pantlingiana W.W. Sm. & - & upto 3600 & $1,2,13$ & Ind $\mathrm{Or}$ & Indian Himalaya (Sikkim) & Rare & - \\
\hline **S. piptathera Edgew. & $\begin{array}{l}\text { Himalayan } \\
\text { Saw-wort }\end{array}$ & $3200-4600$ & 2,3 & Reg Himal & $\begin{array}{l}\text { India (Himachal Pradesh, } \\
\text { Lahaul, Koksar, Tehri- } \\
\text { Garhwal, Sikkim), Nepal }\end{array}$ & $\mathrm{Co}_{0}$ & - \\
\hline **S. polystichoides Hk. & - & $4200-4800$ & $1,2,11$ & Reg Himal & C Nepal, India (Sikkim) & Occ & - \\
\hline S. rufinervis DC. & - & $>2500$ & $1,2,11,13$ & Corea & $\begin{array}{l}\text { W Himalaya } \\
\text { (Himachal Pradesh) }\end{array}$ & Rare & - \\
\hline${ }^{* * S}$ roylei (DC.) Sch.-Bip. & - & $3000-4200$ & $2,3,4,7,9$ & Reg Himal & $\begin{array}{l}\text { India (Uttar Pradesh, } \\
\text { Uttar Kashi, Bhojwasa, } \\
\text { Himachal Pradesh, Kashmir) } \\
\text { Nepal }\end{array}$ & Rare & $\begin{array}{l}\text { The entire plant is used in Tibetan } \\
\text { medicine. It has a bitter taste and a } \\
\text { cooling potency. Antidote, anti- } \\
\text { inflammatory, emollient, hemostatic } \\
\text { and vasoconstrictor, it is used } \\
\text { in the treatment of wounds, excessive } \\
\text { bleeding and meat poisoning. } \\
\text { A paste of the plant is used as } \\
\text { poultice to relieve aching joints. }\end{array}$ \\
\hline
\end{tabular}


Table 1. Continued from previous page.

\begin{tabular}{|c|c|c|c|c|c|c|c|}
\hline Taxa & LNVN & $\begin{array}{l}\text { Altitudinal } \\
\text { range (m) }\end{array}$ & Habitat(s) & Nativity & Distribution & Status & Indigenous uses \\
\hline S. schultzii Hk. & - & $>3000$ & 5,10 & Reg Himal & $\begin{array}{l}\text { China, India } \\
\text { (Kashmir, Ladakh), } \\
\text { N Pakistan }\end{array}$ & Rare & Medicinal \\
\hline $\begin{array}{l}\text { S. simpsoniana } \\
\text { (Field \& Gard.) Lipsch. }\end{array}$ & $\begin{array}{l}\text { Jogi Badshah, } \\
\text { Ghuggi, Yogesh } \\
\text { Fen Kamal }\end{array}$ & $\begin{array}{l}3300-5600 \\
\text { awar, }\end{array}$ & $2,8,9,10,13$ & Reg Himal & $\begin{array}{l}\text { China, Tibet, India } \\
\text { (Uttar Pradesh, Chamoli } \\
\text { Garhwal, Hemkund, Kashmir, } \\
\text { Sikkim, Himachal Pradesh), } \\
\text { Bhutan, Nepal, Pakistan }\end{array}$ & EN & $\begin{array}{l}\text { The plant is used for all kinds of } \\
\text { nervous debility; root extract for } \\
\text { snake bite, plague and painful } \\
\text { periods; roots and essential oil as } \\
\text { insecticides. Also useful for cough, } \\
\text { blood purification, leucorrhea and } \\
\text { sexual problems. }\end{array}$ \\
\hline S. stella Maxim. & - & $4500-4800$ & $1,2,11$ & China & $\begin{array}{l}\text { China, India (Sikkim), } \\
\text { Bhutan, SE Tibet }\end{array}$ & Rare & Medicinal \\
\hline S. stoliczkae Cl. & - & $>2500$ & $1,2,11$ & Reg Himal & $\begin{array}{l}\text { India (Kashmir, Himachal } \\
\text { Pradesh), Nepal, Tibet, } \\
\text { N Pakistan, China }\end{array}$ & Rare & - \\
\hline $\begin{array}{l}\text { S. stracheyana } \\
\text { (Kuntze) Lipsch. }\end{array}$ & - & $2600-3800$ & $1,2,3,4,9$ & Sibir & $\begin{array}{l}\text { India (Uttar Pradesh, Uttar } \\
\text { Kashi, Harki Dun, Kashmir), } \\
\text { Nepal }\end{array}$ & Rare & \\
\hline S. subulata $\mathrm{Cl}$. & - & $4100-5300$ & $2,4,13,15$ & Reg Himal & $\begin{array}{l}\text { Tibet, India (Sikkim), } \\
\text { N Pakistan, China }\end{array}$ & Rare & - \\
\hline${ }^{*}$ S. sudhanshui Hajra & - & $2000-3000$ & 8,11 & Ind & India (Uttar Pradesh) & Rare & Medicinal \\
\hline **S. sughoo Cl. & - & $3300-4800$ & $1,2,13$ & Ind $\mathrm{Or}$ & CE Nepal, India (Sikkim) & Rare & Medicinal \\
\hline **S. taraxacifolia Wall. ex DC. & - & $3000-4500$ & $2,5,9,11,13$ & Reg Himal & $\begin{array}{l}\text { Bhutan, India (Uttar } \\
\text { Pradesh, Chamoli Garhwal, } \\
\text { Keal Bhairawn, Kashmir, } \\
\text { Sikkim), Nepal, Pakistan }\end{array}$ & Occ & Used for ulcer and cold \\
\hline${ }^{* *}$ S. thomsonii $\mathrm{Cl}$. & - & $4000-5200$ & 2 & Reg Himal & $\begin{array}{l}\text { India (Kashmir), } \\
\text { N Pakistan, Tibet }\end{array}$ & Rare & - \\
\hline S. thoroldii Hemsley & - & $3100-5200$ & 14,15 & Reg Himal & $\begin{array}{l}\text { India (Kashmir), } \\
\text { N Pakistan, Tibet, China }\end{array}$ & Rare & - \\
\hline${ }^{* *}$ S. tridactyla Sch.-Bip. & Snow Lotus & upto 5100 & $2,4,5,8,9,10,13$ & Reg Himal & $\begin{array}{l}\text { India (Sikkim), Nepal, } \\
\text { Bhutan, Tibet }\end{array}$ & Rare & Ornamental \\
\hline $\begin{array}{l}\text { S. uniflora (DC.) } \\
\text { Wall. ex. Sch.-Bip. }\end{array}$ & - & $3300-4200$ & $2,9,11$ & Reg Himal & $\begin{array}{l}\text { India (Sikkim, Lachung), } \\
\text { Nepal, Bhutan, SW China }\end{array}$ & Rare & - \\
\hline S. wernerioides Sch.-Bip. ex. Hk. & - & $4500-4950$ & $2,5,9,11,13$ & Reg Himal & $\begin{array}{l}\text { India (Sikkim), Nepal, } \\
\text { Bhutan, SE Tibet, SW China }\end{array}$ & Rare & - \\
\hline **S. yakla Cl. & - & $3600-4800$ & $2,9,11,13$ & Reg Himal & $\begin{array}{l}\text { India (Sikkim), Nepal, } \\
\text { Bhutan, Tibet (SE Lhasa) }\end{array}$ & Rare & - \\
\hline
\end{tabular}

LN=Local Names; VN=Vernacular Names; ${ }^{*}=$ Endemic; **=Near Endemic; $1=$ Shady moist; $2=$ =Alpine meadow/slopes; $3=$ Shrubberies; $4=0$ pen grassy slope; $5=$ Shady rock/boulders; $6=$ Roadside/waste places; $7=$ Cultivated area/Agricultural fields; $8=$ Dry places; $9=$ Rocky slopes; $10=$ Snowline/glacier slopes; $11=$ Forest; $12=$ Riverine; $13=$ =Alpine screes; $14=F l o o d e d$ area; $15=$ Saline alkali lands; Reg Himal=Himalayan Region; CR=Critically endangered; EN=Endangered; $\mathrm{Co}_{0}=$ Common; Unco=Uncommon; Occ=0ccasional; Ind=India; Or=Oriental; Trop=Tropical; As=Asia; Turk=Turkestan; Mont=Mountain; Kara=Karakorum; Sibir=Siberia; Afghan=Afghanistan; Occi=0ccidentalis; N=North; S=South; $\mathrm{E}=\mathrm{East} ; \mathrm{W}=$ West; $\mathrm{C}=$ Central

tion to generation. Therefore, the documentation of indigenous uses is essential for defining priorities for conservation of the species. Recorded information on the economic value of the species of Saussurea is very fragmentary.

In the present study, of the total species examined, indigenous uses of 28 species were known, and of these, 27 species had medicinal value or are used for the treatment of various diseases and ailments (Table 1). Six species ( $S$. affinis, $S$. auriculata, S. bracteata, $S$. costus, $S$. gossypiphora and $S$. obvallata) are used as medicine, food, fodder, fuel, and for ornamental and religious purposes. Among the various parts, i.e. leaves, stem, flowers, seeds, aerial parts, roots, etc. of the plants used for curing diseases, roots are the most often cited. All parts of the plant of $S$. ceratocarpa, $S$. costus, $S$. graminifolia, $S$. medusa, $S$. obvallata and $S$. roylei are said to be used for medicinal purposes.

Among the species of Saussurea, $S$. costus is the most commercially viable species. Its medicinal properties are well documented in traditional Chinese medicine, the Tibetan system of medicine, and ayurvedic medicine. Out of the 175 formulations reported in The Handbook of Traditional Tibetan Drugs, this species is one of the main ingredients in 71 formulations. ${ }^{45}$ The roots of $S$. costus have a strong and sweet aromatic odor with a bitter taste, and are used as an antiseptic and in controling bronchial asthma, particularly of the vagotonic type. $^{8}$

Preparations made from this species are also reported to cure various diseases and conditions including dysentery, rheumatism, bronchitis, cholera, jaundice, ulcers, skin diseases, stomachache, gas, toothache, cough, cold, fever, edema, bruises and cuts. ${ }^{8}$ The oil extracted from the roots is known as Costus Oil, which is used in high-grade perfumes and in the preparation of hair oil. Costus Oil is also said to be effective in the treatment of leprosy. In the Himalayan states of India, the roots are used as insecticide to protect shawls and woollen fabrics, and as incense. In the Lahaul and Spiti districts of Himachal Pradesh, dried leaves of Kuth are smoked as tobacco and 
upper parts of its plants are used as fuel and fodder. ${ }^{8,42}$ The roots of $S$. auriculata can be substituted for the roots of $S$. costus. ${ }^{8}$

Besides $S$. costus, $S$. obvallata and $S$. gossypiphora are other high value species used not only for medicine, but also highly valued for religious purposes. ${ }^{8,46}$ These species are offered for worship at hill shrines and used to guard against evil spirits. The former, known as 'Brahma Kamal' has been designated the state flower for Uttarakhand. It is offered to the goddess 'Nanda Devi' and other deities on auspicious days. Some species such as $S$. bracteata, S. gnaphaloides, S. costus, S. obvallata and $S$. taraxacifolia are used as the main ingredients in the 'Amchi' Medical System, a traditional system of Tibetan medicine. ${ }^{47}$ Besides indigenous uses, the roots of $S$. costus, $S$. gossypiphora and $S$. simpsoniana yield an essential oil which has been sold for a premium in making high-grade perfume, various medicines and insecticides.

\section{IUCN status}

Habitat specificity, population size, distribution range and anthropogenic pressure play an important role in identifying the status of the species. ${ }^{48}$ Being habitat specific and distributed in a narrow geographical range, most high altitudinal species of Saussurea in the Himalayas are in need of some sort of protection (conservation)..$^{49}$

Of the total species identified in the present study, 44 were identified as rare, 2 species, $S$. costus and $S$. gossypiphora, as critically endangered, and 2 species, S. obvallata and S. simpsoniana, as endangered, due to heavy pressure on these species. Other species were identified as occasional, uncommon and common in the study region (Figure 3 ). Population assessment using standard ecological methods is suggested for actual quantification of existing stock of the rare and threatened species in natural habitats.

$S$. costus has been in high demand in the pharmaceutical industry, but during the last decade the species has been even more popularized due to its threatened status globally. Due to high market demand and uncontrolled exploitation of the species, it was reported to be extinct in many pockets in the wild. ${ }^{4}$ To meet the market demand and conserve the species, commercial cultivation of $S$. costus was taken up in the neighboring villages of the habitats in Kashmir, and subsequently it was initiated in high altitude areas of neighboring states such as Himachal Pradesh and Uttarakhand. Being an endemic species to the Himalayas, the distribution of $S$. costus is quite restricted to an extremely narrow geographical range. ${ }^{50}$

Due to great demand for raw material from these plants, most of the natural populations of the species are either under destructive har-

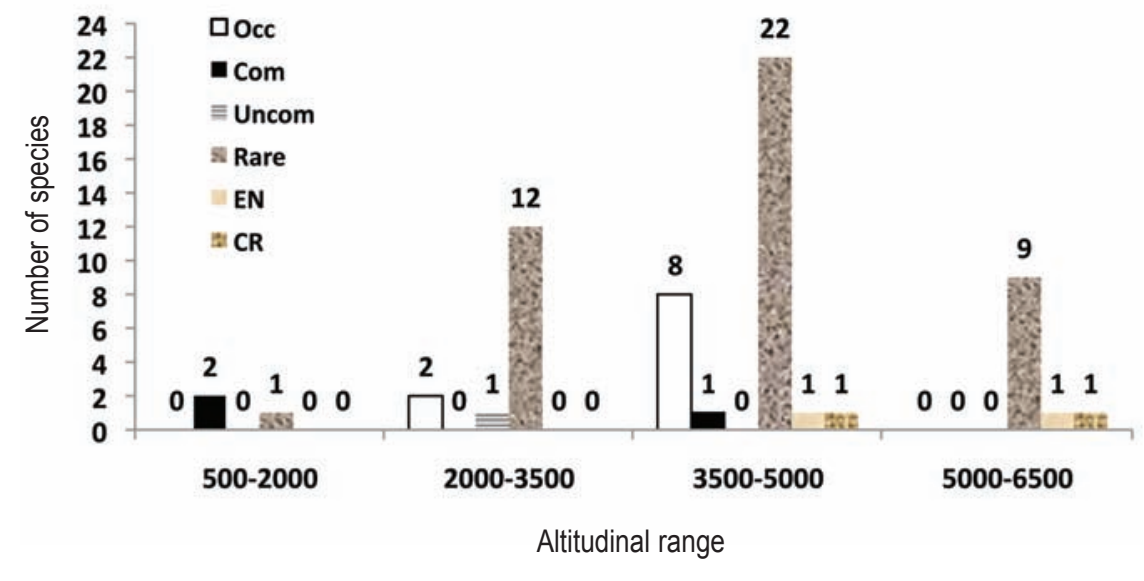

Figure 3. IUCN status of Saussurea species distributed in different altitudinal ranges (Abbreviations used: Occ=Occasional; $\mathrm{Com}=$ Common; Uncom=Uncommon; EN=Endangered; CR=Critically Endangered)

vesting or have been extirpated. Consequently, this invaluable species has been declared endangered $^{51,52}$ and is listed in Appendix $I$ of CITES (Convention of International Trade in Endangered Species of Wild Fauna and Flora). The species is also included in the negative list of exports of the Ministry of Commerce, Government of India and in the 'Schedule VI' of the Wildlife Protection Act of India. Also, trade of the species is strictly prohibited under the Foreign Trade Development Act-1992. This species is being cultivated commercially in the cold desert of Lahaul Valley, but owing to the fluctuating and low market prices of raw material, the cultivation is restricted to a few villages. ${ }^{42}$

Besides S. costus, many other species of Saussurea are categorized under rare and threatened. Apart from the restricted distribution, the harvesting of whole plants of most of the species of Saussurea is one of the reasons for their threatened status (harvesting of plant parts which permit regeneration or re-growth is a less damaging mode of use). Incidentally, $S$. ceratocarpa, $S$. graminifolia, $S$. medusa, $S$. obvallata and $S$. roylei are the prominent species whose whole plant is used for medicine and thus, these species are more prone to extinction and need immediate conservation. Law and Salick ${ }^{53}$ revealed that the plants of $S$. laniceps (found in the Himalayan region), being larger in size, are easier to find and moreover, the whole plant of this species is harvested just before seed set. Therefore, early or untimely collection of the plants may also be one of the threats to the species.

\section{Review of propagation and cultivation}

The review of literature indicates that the propagation (conventional and in vitro) and cultivation techniques are lacking for most of the species of Saussurea excluding $S$. costus, ${ }^{28,34}$ S. obvallata ${ }^{35,36}$ and $S$. medusa ${ }^{37,38}$ Cultivation of
S. costus in the Cold Desert of the Lahaul valley was initiated during the 1920 s, and at present the species is being cultivated commercially. ${ }^{8,42}$ Small scale cultivation of the species by some tribal communities has also been reported from the Nanda Devi Biosphere Reserve (2000-3500 m) of the Uttarakhand ${ }^{39}$ In S. laniceps, due to its high medicinal and edible value and rarity, efforts are being made in Tibet towards its conservation and cultivation. ${ }^{40}$ Information on the propagation and cultivation of some species of Saussurea is available at the Rock Garden Plant Database website. ${ }^{41}$

\section{Conclusions}

The present study provides comprehensive information on the diversity, distribution, habitat preference, nativity, endemism and status of 62 species of Saussurea in the IHR. Among these, indigenous uses of 28 species are known in the region. The genus Saussurea shows high habitat specificity as 16 species were recorded to be restricted to one or two habitats only. A few of the species are known for their economic value and, therefore, other species should also be investigated for their economic importance. $S$. affinis, $S$. auriculata, S. bracteata, $S$. costus, $S$. gossypiphora and $S$. obvallata were recorded as multipurpose species, used as medicine, food, fodder, fuel, and for ornamental and religious purposes. Considering the high industrial demand for raw material and the endangered status of $S$. costus, S. gossypiphora, S. obvallata and $S$. simpsoniana, these 4 species should be priorities for conservation (in situ and ex situ) throughout the IHR. Population assessment of the native, endemic and rare/endangered species using standard ecological methods has been suggested for the quantification of the 
existing stock of these species in their natural habitats. Phytochemical investigations for the identification of active ingredients are urgently required to identify the potential of the species. A review of the literature indicates that propagation and cultivation techniques are available only for a few species of Saussurea and, therefore, these techniques need to be developed, particularly for rare/ endangered and multipurpose species. Native communities need to be made aware of the sustainable use and conservation value of the species of Saussurea.

\section{References}

1. Singh DK, Hajra PK. Floristic diversity. In: Biodiversity status in the Himalaya. Edited by Gujral. British Council, New Delhi, India 1997;23-38.

2. Samant SS, Dhar U, Palni LMS. Medicinal Plants of Indian Himalaya: Diversity, Distribution Potential Values. HIMAVIKAS Publ. No. 13, Gyan. Prakash., Nainital, 1998;163.

3. Hajra PK, Rao RR, Singh DK, Uniyal BP . Flora of India. Vols. 12 \& 13: Asteraceae. Botanical Survey of India, Calcutta, India 1995.

4. Sinha GP, Shukla BK. The Gnaphaleae (Asteraceae) in Sikkim. J Hill Res 1997; 10:261-72.

5. Hajra PK. Bramkamal and its Allies (Saussurea the genus). Jugal Kishore and Co., Dehradun, India 1988.

6. Gaur RD. Flora of the district Garhwal, Northwest Himalaya (with ethnobotanical notes). Trans Media, Srinagar Garhwal, India 1999.

7. Kumar S , Singh V. Asteraceae of Sikkim. Deep Publications, New Delhi, India 2001; 209.

8. Anonymous. The Wealth of India: A Dictionary of Indian Raw Materials and Industrial Products. Council of Scientific and Industrial Research. New Delhi, India 1972;Vol.IX: Rh-So.

9. Anonymous. Asteraceae: Cardueae: Saussurea DC. [Draft], Ann Mus NatlHist. Nat.16: 156, 198. 1810, nom. cons.

10. Collet H. Flora Simlensis, a handbook of the flowering plants of Simla and the neighbourhood. Bishen Singh Mahendra Pal Singh, Dehra Dun 1980;652.

11. Hara H, Chater A0, Williams LHJ. An enumeration of the flowering plants of Nepal, British Library Publication No. 854, Mansell Bookbinders Limited 1982.38-41.

12. Chowdhery HJ, Wadhwa BM. Flora of Himachal Pradesh (Flora of India Series 2). Botanical Survey of India, Vol. 2, Raje Printers, New Delhi 1984;677.
13. Dhar U, Kachroo P. Alpine flora of Kashmir Himalaya. Scientific Publishers, Jodhpur, India 1983.

14. Naithani BD. Flora of Chamoli, Vol I - II. Botanical Survey of India, Howrah, New Delhi 1984.

15. Naithani HB. Flowering plants of India, Nepal and Bhutan. Surya Publishers, Dehradun 1990;p.711.

16. Jain SK. Dictionary of Indian Folk Medicine and Ethnobotany. Deep Publications, New Delhi, India 1991.

17. Aswal BS, BN Mehrotra. Flora of LahaulSpiti (A Cold Desert in North West Himalaya).Bishen Singh Mahendra Pal Singh, Dehradun, India 1994.

18. Samant SS, Joshi HC and Arya SC. Diversity, nativity and endemism of vascular plants in Pindari area of Nanda Devi Biosphere Reserve-II. Himalayan Biosphere Reserves 2000;2:1-29.

19. Samant SS, Joshi HC, Pant S, Arya SC. Diversity, nativity and endemism of vascular plants of Valley of Flowers National Park. Himalayan Biosphere Reserves 2001 3: 1-17.

20. Kala CP, Rawat GS Uniyal VK. Ecology and conservation of the Valley of Flowers National Park, Garhwal Himalaya. Wildlife Institute of India, Dehradun, India 1998.

21. Pangtey YPS, Samant SS, Rawat GS. Contribution to the flora of Pithoragarh district, Kumaon Himalaya. Himalayan Research \& Development 1988;7:24-46.

22. Samant SS. Diversity, nativity and endemism of vascular plants in a part of Nanda Devi Biosphere Reserve in West Himalaya I. Himalayan Biosphere Reserve 1999;1-28.

23. Singh SK, Rawat GS. Flora of Great Himalayan National Park, Himachal Pradesh. Bishen Singh Mahendra Pal Singh, Dehra Dun 2000;305.

24. Saklani A, Rao RR, Chaudhary LB. SEMcharacterisation of achene morphology towards the taxonomy of Indian species of Saussurea DC. (Asteraceae). Rheedea 2000;10:1-18.

25. Joshi HC, Arya SC and Samant SS. Diversity, distribution and indigenous uses of plant species in Pindari area of Nanda Devi Biosphere Reserve-II. Indian J For 2001;24: 514-536.

26. Anonymous. Index Kewensis Plantarum Phanerogamarum Vol. 1-2 (1883-1885) and Suppl. 15 (1886-1970). Clarendron Press, 0xford 1883-1970.

27. Dhar U, Samant SS. Endemic plant diversity in the Indian Himalaya I. Ranunculaceae and Paeoniaceae J Biogeogr 1993;20:659-65.

28. Arora R, Bhojwani SS. In vitro propagation and low temperature storage of Saussurea lappa C.B. Clarke - An endangered, medic- inal plant. Plant Cell Reports 1989;8:44-47.

29. Chauhan JS, Tomar YK, Vashist DP. Effect of various levels of IAA on the seed germination of Saussurea costus (Falc.) Lipschitz (S. lappa (Decne.) Sch.-Bip.). J Indian Bot Soc 1998;77:75-177.

30. Badola HK, Butola JS. Threatened medicinal plant cultivation succeeds in Himachal Himalaya. Conserv Biol. 2004;229:1.

31. Kaushal SK, Rana U. Effect of growth regulators on germination, growth and yield of Kuth (Saussurea lappa Clarke). Indian Journal of Agricultural Research 2004;38: 45-49.

32. Rawat YS, Oinam SS, Vishvakarma SCR, Kuniyal JC. Saussurea costus (Falc.) Lipsch.: A promising medicinal crop under cold desert agro-ecosystem. Indian Journal of Forestry 2004;27:297-303.

33. Sharma RK, Sharma S, Sharma SS. Seed germination behaviour of some medicinal plants of Lahaul and Spiti cold desert (Himachal Pradesh): Implications for conservation and cultivation. Curr Sci 2006; 90:1113-8.

34. Butola JS, Samant SS. Seed viability of Saussurea costus. J Tropical Medicinal Plants 2006;7:97-203.

35. Joshi M , Dhar U. In vitro propagation of Saussurea obvallata (DC.) Edgew-an endagered ethnoreligious medicinal herb of Himalaya. Plant Cell Reports 2003;21: 933-939.

36. Dhar U, Joshi M. Efficient plant regeneration protocol through callus for Saussurea obvallata (DC.) Edgew. (Asteraceae): effect of explant type, age and plant growth regulators. Plant Cell Reports 2005;24:195200.

37. Zhao DX, Qiao CL, Wang Y. Cell culture and selection of high flavonoid production during cell lines of Saussurea medusa. Acta Botanica Sinica 1998;40:515-520.

38. Zhao D, Xing J, Li M, Lu D. Optimazation of growth and jaceosidin production in callus and cell suspension cultures of Saussurea medusa. Plant Cell Tissue and Organ Culture 2001;67:227-34.

39. Maikhuri RK, S Nautiyal, KS Rao and KG Saxena. Medicinal plant cultivation and biosphere reserve management: A case study from Nanda Devi Biosphere Reserve, West Himalaya. Curr Sci 1998;74:157-63.

40. All The Tea Company, Premium Organic Tibetan Tea 2007.

41. Slaby P.. Rock Garden Plants (version 3.0).

42. Kuniyal CP, Rawat YS, Oinam SS, Kuniyal JC, Vishvakarma SCR. Kuth (Saussurea lappa) cultivation in the cold desert environment of the Lahaul valley, northwestern Himalaya, India: arising threats and need to revive socio-economic values. Biodiversity and Conservation 2005;14: 1035-45. 
43. Anonymous. Carrying Capacity study of Teesta Basin in Sikkim. Vol-VI. Biological Environment-Terrestrial and Aquatic Resources. Centre for Inter-Disciplinary Studies of Mountain and Hill Environment. University of Delhi, Delhi 2005;332.

44. Dhar U. Conservation implications of plant endemism in high-altitude Himalaya. Curr Sci 2002;82:141-8.

45. Tsarong TJ. Handbook of Traditional Tibetan drugs, their nomenclature, composition, use and dosage. Tibetan Medical Publications, West Bengal, India 1986;101.

46. Samant SS, Pant S. Diversity, distribution pattern and traditional knowledge of Sacred Plants in Indian Himalayan Region. Indian Journal of Forestry 2003;26:201-13.

47. Kala CP. Medicinal plants of the high altitude cold desert in India: Diversity, distri- bution and traditional uses. International Journal of Biodiversity Science and Management 2006;2:43-56.

48. Samant SS, Dhar U, Rawal RS. Conservation of rare endangered plants: The context of Nanda Devi Biosphere Reserve. In: P.S. Ramakrishnan, A.N. Purohit, K.G. Saxena, K.S. Rao \& R.K. Maikhuri (eds.), Conservation and Management of Biological Resources in Himalaya. Oxford \& IBH Publishing Company Private Limited, New Delhi. 1996;521-545.

49. Klein R. Phytoecdysteroids. Journal of the American Herbalists Guild, Fall/Winter issue 2004.

50. Siddique MAA, Wafai BA, Riya AM, Sheikh AS. Conservation of Kuth (Saussurea costus). A threatened medicinal plant of Kashmir Himalaya. In: Samant, S.S., Dhar,
U. and Palni, L.M.S. (eds.), Himalayan Medicinal Plants: Potential and Prospects, 2001:197-204., HIMAVIKAS, occasional publication No. 14, Gyan. Prakash., Nainital.

51. Anonymous. Convention on International Trade in Endangered Species of Wild Fauna and Flora. Signed at Washington, DC on 3 March, 1973 and amended at Bonn, Germany on 22 June 1979.

52. Nayar MP, Shastry ARK. Red Data Book of Indian Plants. Three volumes. Botanical Survey of India, Kolkata, India 1987, 1988 and 1990.

53. Law W, Salick J. Human-induced dwarfing of Himalayan snow lotus, Saussurea laniceps (Asteraceae). Proceeding of the National Academy of Sciences of the USA. 2005;102:29,0218-10220. 